\title{
Efficient Aerial-Aquatic Locomotion with a Single Propulsion System
}

\author{
Yu Herng Tan ${ }^{1}$, Rob Siddall ${ }^{1}$ and Mirko Kovac ${ }^{1}$
}

\begin{abstract}
Aerial-Aquatic locomotion would allow a broad array of tasks in robot enabled environmental monitoring or disaster management. One of the most significant challenges of aerial-aquatic locomotion in mobile robots is finding a propulsion system that is capable of working effectively in both fluids, and transitioning between them. The large differences in the density and viscosity of air compared to water means that a single direct propulsion system without adaptability will be inefficient in at least one medium. This paper examines multimodal propeller propulsion using computational tools validated against experimental data. Based on this analysis we present a novel gearbox enabling an aerial propulsion system to operate efficiently underwater. This is achieved with minimal complexity using a single fixed pitch propeller system, which can change gear underwater by reversing the drive motor, but with the gearing arranged to leave the propeller direction unchanged. This system is then integrated into a small robot, and flights in air and locomotion underwater are demonstrated.
\end{abstract}

Index Terms-Mechanism Design, Micro/Nano Robots, Biologically-Inspired Robots, Aerial Systems: Mechanics and Control, Field Robots

\section{INTRODUCTION}

$\mathbf{L}$ OCOMOTION in unstructured terrain is a significant challenge to miniature robots, often requiring operation in water, air and on the ground. In particular, aerial-aquatic robots face major challenges, and must accommodate the increased structural loads, fluid inertia and drag encountered underwater, without compromising the weight and lifting area requirements of flight.

Addressing these challenges would allow unique robot operation in a wide variety of environments, such as tidepools, wetlands or canal systems, enabling autonomous monitoring of contaminants and ecosystem health. To achieve this, we are developing a novel robot, called the Aquatic Micro Air Vehicle (AquaMAV) [1] which is capable of aerial and aquatic locomotion. The AquaMAV will be able to dive directly into the water at high speeds to achieve initial depth, subsequently retaking flight using a high powered burst of thrust (figure 1), as demonstrated in [2]. But while able to escape water, this robot had no means of propelling itself underwater. To add aquatic locomotion it is attractive to use

Manuscript received: September 10 2016; Revised December 7 2016; Accepted January 192017.

This paper was recommended for publication by Editor Jonathan Roberts upon evaluation of the Associate Editor and Reviewers' comments.

1 Department of Aeronautics, Imperial College London, London, SW7 2AZ, UK. Correspondence should be directed to r.siddalleimperial.ac.uk A video attachment to this paper is available online: tinyurl.com/AerialAquaticPropulsion

Digital Object Identifier (DOI): see top of this page.

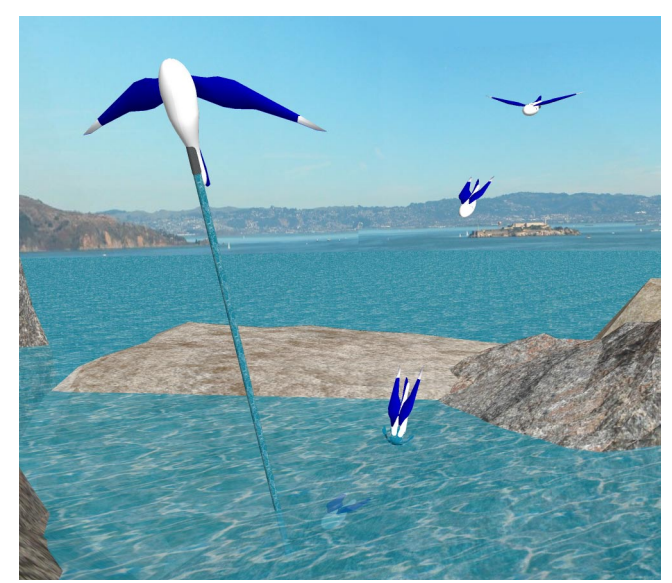

Fig. 1: Concept sketch of an Aquatic Micro Aerial Vehicle diving into the water, gathering data and retaking flight.

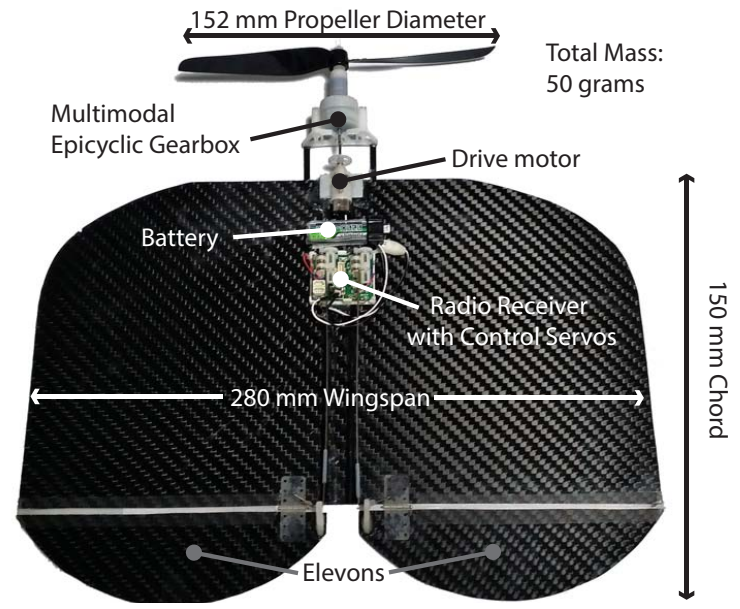

Fig. 2: Miniature 50 gram aircraft which uses a dual mode gearbox to achieve energetically efficient aerial-aquatic locomotion with a single propeller.

the same propulsion system as is used for flight, as this reduces the weight and complexity of the system. However, the increase in load a propeller in a denser fluid results in much slower rotation speeds, and means a significant loss of motor efficiency.

In this paper, we will examine the efficiency of propeller operation in air and water, using a blade element code (QPROP [3]) to investigate a variety of propeller geometries commonly used in Micro Aerial Vehicle (MAV) propulsion. These theoretical predictions are then validated against un- 
derwater thrust tests, and the model is used to investigate an ideal aerial-aquatic transmission. Based on the results of these investigations, we propose a bimodal epicyclic transmission to allow efficient propeller operation in air and water. This gearbox is then fabricated and efficient propulsion of a miniature aircraft (figure 2) in air and water is demonstrated. By using the direction of the motor to control the setting of the gearbox, the proposed design provides a simple and effective solution to achieving efficient aerial-aquatic locomotion. Compared to previous designs for multimodal operation in air and water, this system significantly improves the overall performance of the propulsion system in the two media, with minimal mechanical complexity and no additional actuators.

\section{Existing AERiAl-Aquatic Robots}

Although the development of fully functional aerialaquatic robots is still in its early stages, several prototypes have demonstrated multimodal abilities to varying degrees [4], [5]. A summary of developments presented in [6] classifies the types of aerial-aquatic vehicles according to the degree of autonomy and operation functions. There exists an abundance of vehicles with mission profiles that include contact with water, such as seaplanes with surface take-off capabilities or submarine-launched UAVs. However, neither of these classes of vehicles are capable of self-propulsion underwater. Seaplane-type vehicles are too buoyant to submerge themselves and submarine-launched UAVs are typically assisted by discardable support systems underwater, before they transition to the air.

Producing an aerial-aquatic system which is capable of active propulsion in both media has proved challenging, with few successful prototypes presented in recent years. Multiple studies have investigated the possibility of aerial-aquatic locomotion with a single propulsor, a preferred approach since weight has a major impact on aerial performance. [7] and [8] investigated the use of flapping foils for dual mode propulsion, using changes in morphology and kinematics to ensure efficient thrust production in different media. Both [7] and [8] demonstrated the efficacy of this strategy in tunnel tests. On a much smaller platform, [9] demonstrated aerialaquatic locomotion with an insect scale vehicle. This system used flapping wings for both aerial and aquatic propulsion, with changes in stroke rate resulting in dynamically similar flows in both media. However, the robot was powered externally so energetic efficiency was not of immediate concern.

Recently, quadrotor platforms with hybrid aerial-aquatic capabilities have been demonstrated, using aerial propellers for locomotion underwater [10], [11]. [10] uses buoyancy control to transition to a submersible mode, while [11] demonstrates direct air-water transition using a dual-propeller system. Both robots use the same motor-propeller combination in air and water. Although both demonstrated the ability to move underwater, the use of aerial propulsion system in off-design conditions results in the system operating at very low efficiencies.

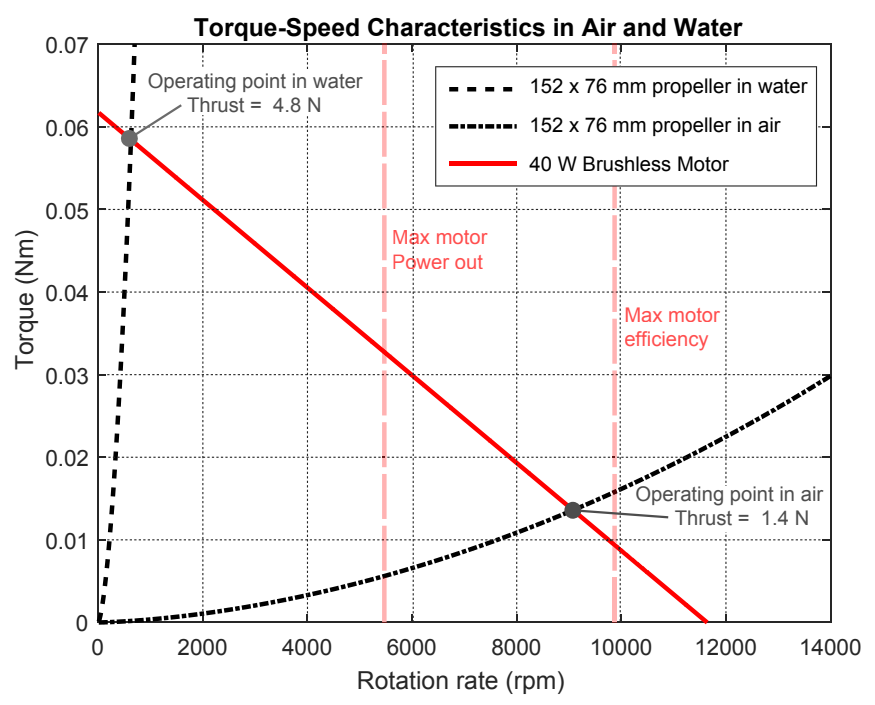

Fig. 3: Torque speed curves for a $152 \mathrm{~mm}$ diameter, 76 $\mathrm{mm}$ pitch propeller in air and water and a $40 \mathrm{~W}$ example brushless motor. We have marked the rotation rate at which the motor operates at maximum efficiency $\left(\eta_{M}\right.$, equation 2) and produces maximum output power $(Q \omega)$ with dashed lines. The torque-speed curve of the propeller shifts steeply to the left in water, increasing equilibrium torque and decreasing operating rpm. In air, the propeller operates at near maximum motor efficiency, but in water the motor is at only $6 \%$ of its peak efficiency speed.

\section{COMPUTATIONAL INVESTIGATIONS}

The differences between electric propeller operation in air and water were first investigated by analysing motor-propeller combinations using QPROP, examining the effect of propeller geometry, motor parameters and operating conditions on the thrust production and efficiency of the overall system. QPROP computes the steady-state behaviour of propeller systems using an enhanced blade-element and vortex method built on the method of Larrabee [12]. It computes an accurate propeller aerodynamic model by accounting for the propeller's self-induction.

The motor-propeller system is defined by three files containing operating conditions, motor properties, and propeller geometry: The motor is described by a linear model (defined by the zero-load current, internal resistance and characteristic $\mathrm{rpm} / \mathrm{V}$ ) while the propeller is defined geometrically using a series of chord and twist values along the blade, and aerodynamically by section lift and drag coefficients. The simulation's fidelity is then principally limited by the accuracy of propeller geometry and aerodynamic coefficients.

The propellers used in this study are commercially available components, commonly used in small scale aerial robots. We refer to these props as, for example ' $152 \times 76 \mathrm{~mm}$ ' referring to a propeller with a $152 \mathrm{~mm}$ diameter and a $76 \mathrm{~mm}$ blade pitch. The propellers tested in section IV were modelled using a 3D laser scanner (FARO Edge ScanArm, 25 $\mu \mathrm{m}$ accuracy) to generate surface point clouds (see section IV, figure 5B). These were then parsed into QPROP input files using Matlab. 

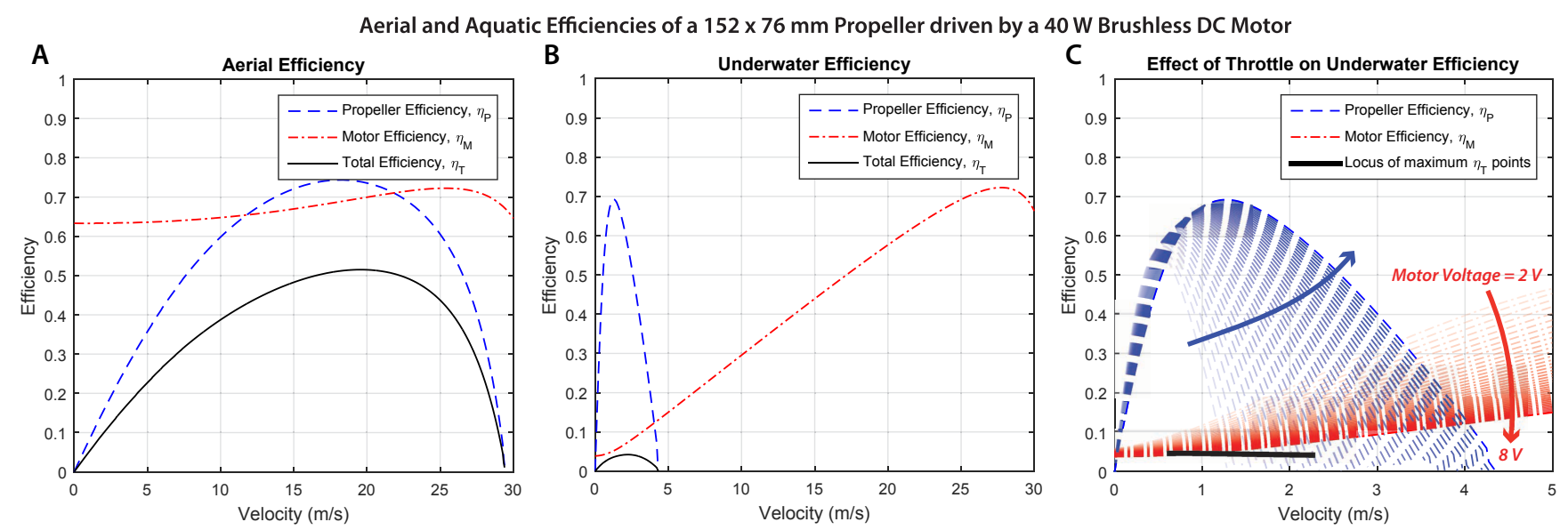

Fig. 4: Efficiencies curves of the same motor-propeller system in air (A) and in water (B) showing that the properties of the medium greatly affect the matching of the system. Efficiency curves shown are for the coupled motor-propeller, such that the forces produced by the propeller effect the operating speed of the motor and vice versa. Beyond a certain forward speed $\eta_{P}<0$ and the propeller is creating drag. (C) Shows the effect of throttling the motor on underwater efficiency by increasing the applied voltage from $2 \mathrm{~V}$ to $8 \mathrm{~V}$. The locus of maximum total efficiency points is marked, showing that throttling has little effect on total efficiency for a mismatched system. Efficiency curves at lower voltages are shown with increased transparency.

A cylinder is fitted to the central hub propeller hub to locate the blade's rotation axis, and cross sections are taken of the blade scan at 30 equally spaced sections, from which chord length, pitch angle and airfoil sections can be extracted for input into QPROP.

Our objective in this investigation is to maximise the efficiency of a propeller propulsion system, achieved by matching propeller and motor efficiencies. Propeller efficiency is defined by the ratio of propulsive power out (thrust, $T$ multiplied by forward speed, $v$ ) to shaft power in (shaft torque, $Q$ multiplied by angular speed, $\omega$ ),

$$
\eta_{P}=T v / Q \omega
$$

and motor efficiency is the ratio of shaft power to electrical power:

$$
\eta_{M}=Q \omega / V I
$$

where $V$ and $I$ are the input voltage and current. Total system efficiency is then:

$$
\eta_{T}=\eta_{P} \eta_{M}=T v / V I=P_{o} / P_{i}
$$

where $P_{i}$ and $P_{o}$ denote input and output power. Efficiency is therefore zero when static and $v=0$, and is strongly a function of forward velocity, which determines the relative motion of propeller blades to the surrounding fluid.

However, to highlight the problems of operating aerial propellers underwater, we have first analysed the torque requirements of a static $152 \times 76 \mathrm{~mm}$ propeller in air and water, driven by a 10 gram brushless motor currently used for aerial propulsion in an AquaMAV prototype [13]. The motor has a peak output power of $40 \mathrm{~W}$, and an unloaded speed of 2000 $\mathrm{rpm} / \mathrm{V}$. Figure 3 shows propeller torque against rotation speed in water and in air, and the torque-speed characteristic of the case study motor. The motor characteristic is given by a first order model of the motor:

$$
Q=\left(\left(V-\frac{\omega}{K_{v}}\right) \frac{1}{R}-I_{0}\right) \frac{1}{K_{v}}
$$

where the relation between the shaft torque, $Q$ and rotational speed, $\omega$, is given by voltage, internal resistance $(R)$, noload current $\left(I_{0}\right)$ and $\mathrm{rpm} / \mathrm{V}\left(K_{v}\right)$, the latter three being characteristic values of a given motor. The rotation speed at which a motor produces maximum output power $(Q \omega)$ and maximum efficiency $\left(\eta_{m}\right.$, equation 2 2 can be calculated analytically, and are marked on figure 3 . When the motor output is connected to a propeller, the operating point of the motor-propeller system is at the intersection of the motor and propeller torque characteristics (figure 3). Here it can be seen that the increased fluid density in water shifts the propeller characteristic backward significantly, forcing the system to operate at a much lower rpm. Here, reduction in speed means that the two propeller flows are dynamically similar, and blade tip Reynolds numbers,

$$
R e=\rho \omega D^{2} / \mu
$$

where $\rho$ is the fluid density, $\mu$ viscosity and $D$ propeller diameter, are $1.08 \times 10^{6}$ and $1.20 \times 10^{6}$ in air and water respectively. However, the thrust produced in water is $4.8 \mathrm{~N}$, over three times the thrust produced in air $(1.4 \mathrm{~N})$. So while the motor can produce significantly more force underwater, it must do so at $10 \%$ of its maximum power output speed, and $6 \%$ of its maximum efficiency speed.

The matching problem of aerial aquatic operation can be seen more clearly when forward velocity is taken into account. In figure $4 \mathrm{~A}$ and $4 \mathrm{~B}$ the efficiencies of the same 

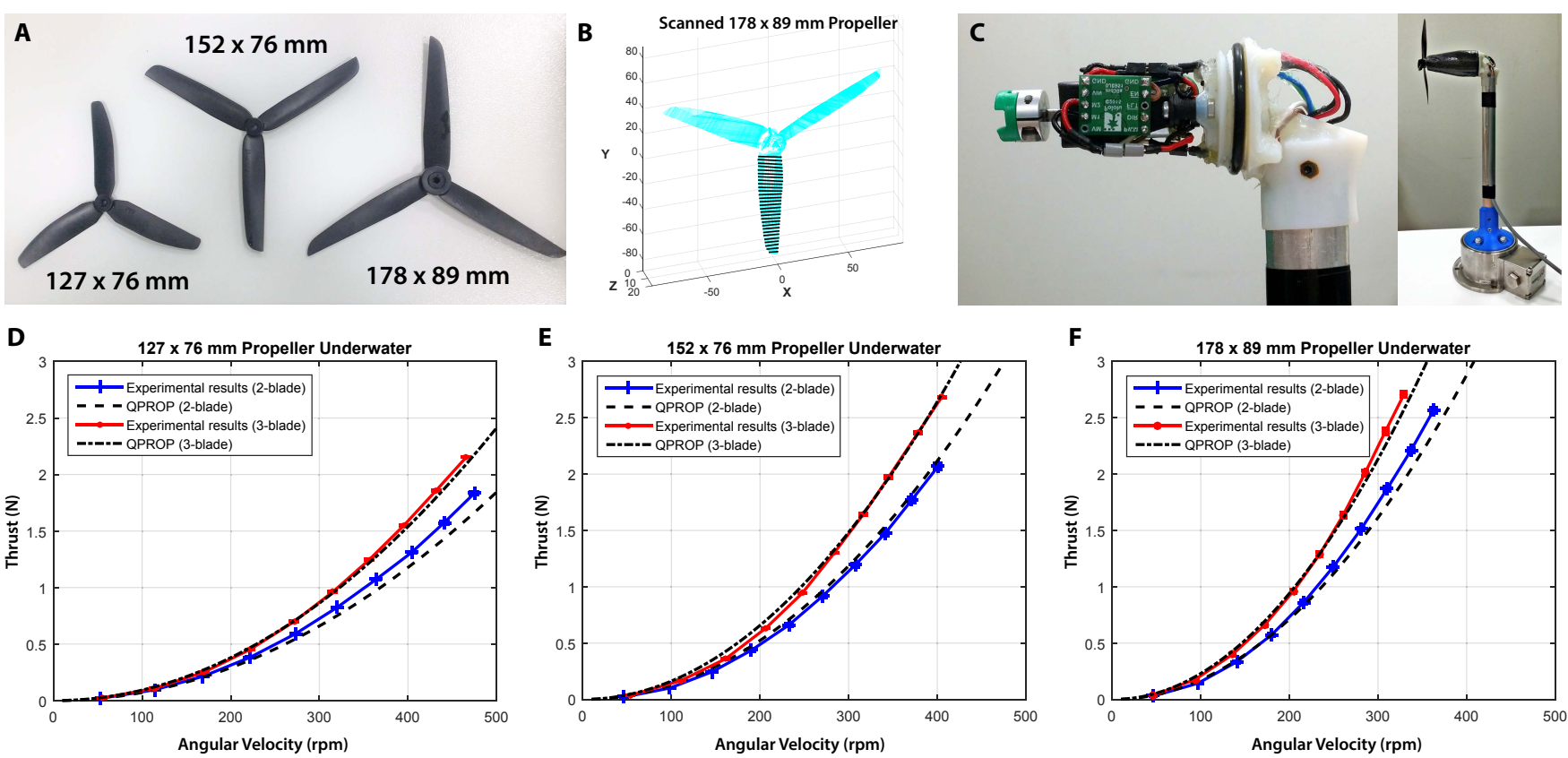

E

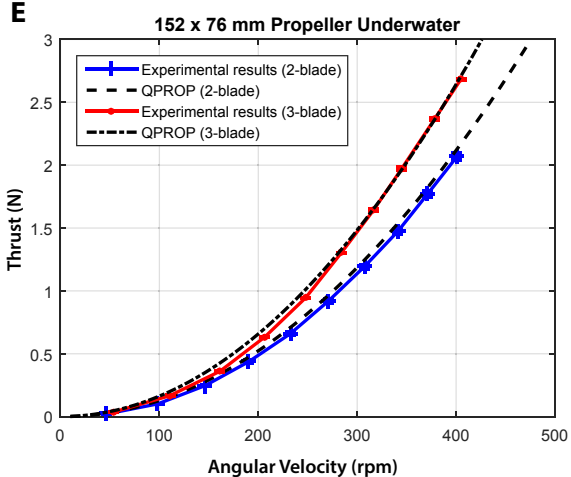

$\mathbf{F}$

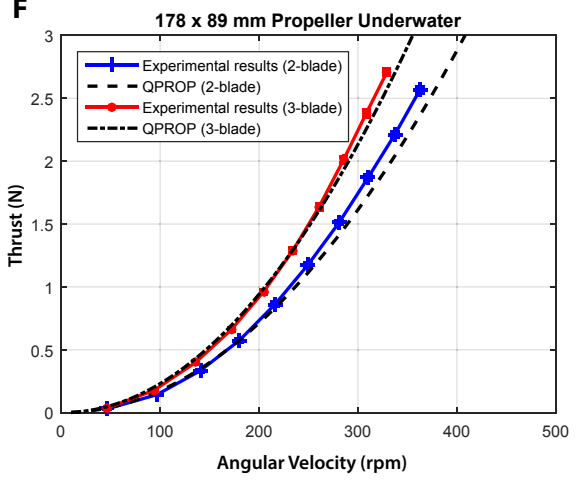

Fig. 5: Thrust tests: A: 3-blade variants of propellers tested. B: Point cloud from 3D scan of propeller geometry. C: Experimental set up, showing motor and electronics with case removed, and full assembly mounted to a waterproof force sensor. D-F: Experiments results for six different aerial propellers used underwater, compared with QPROP predictions, with error bars shown on measured data. Two and three blade variants of three different propeller sizes were tested. Propellers matched well to simulation.

motor-propeller combination are plotted based on QPROP output data over a range of forward velocities in air and water. Beyond a certain speed, the propeller cannot rotate quickly enough, the propeller creates drag rather than positive thrust, and $\eta_{P}<0$. It can be seen that while maximal propeller efficiency is not greatly reduced in water $\left(\eta_{P}=\right.$ $69 \%$ compared to $75 \%$ in air), the motor speed is much lower, forcing the system to operate at a very low efficiency ( $\eta_{T}=5 \%$ compared to $51 \%$ in air). This highlights the key problem in using an aerial propulsion system underwater; it is difficult to achieve good motor-propeller matching in both media. Conversely, if the motor used has a torque characteristic appropriate to the higher resistance in water, the low maximum rpm will result in negligible thrust in air.

The curves shown in figure 4A and $4 \mathrm{~B}$ show the motor operating at full power. When operating at lower power, the efficiency of the system is expected to increase. This is because the equilibrium torque of the system increases with the voltage applied, increasing the mismatch between the motor design torque and required torque. In figure $4 \mathrm{C}$, the effect of changing the motor supply voltage on efficiency is shown. The motor is simulated operating from $2 \mathrm{~V}$ to $8 \mathrm{~V}$, and the maximum total efficiency at each voltage is calculated. The locus of these points is plotted in figure $4 \mathrm{C}$. Propeller and motor efficiency curves are also shown for each simulated voltage, plotted with decreasing transparency as motor voltage is increased. The results show a small but insignificant increase in total efficiency as voltage is reduced (from $4.2 \%$ at $2 \mathrm{~V}$ to $4.6 \%$ at $8 \mathrm{~V}$ ). This shows that although the vehicle will not necessarily always operate at full throttle when travelling, reducing motor power has little effect on total system efficiency.

\section{EXPERIMENTAL VERIFICATION}

In order to verify the computational predictions made using QPROP, several propellers were tested underwater (examples shown in figure 5A) and torque-speed curves were recorded, using encoders (Pololu 12CPR Magnetic Encoder) and a 6-axis force balance (ATI Gamma IP68). Because of the dynamic similarity of the flows in air and water (section III), testing in water was deemed sufficient to validate prediction in both media. In addition, testing in water has advantages over testing in air, as the slower rotation speeds and larger forces in water can be measured more accurately with sensors.

During testing, propellers were driven by a $10 \mathrm{~W}$ brushed gearmotor with a 50:1 gearbox (Pololu 50:1 HPCB6V), which could provide the torque necessary to drive the propellers underwater, without drawing damaging current loads (QPROP simulations showed a large current draw beyond the limits of safe operation when the brushless outrunner described in section III was used underwater).

The encoder mounted to the back of the motor was used to measure rotational speeds of the motor shaft, with an accuracy of 600 pulses per revolution of the output shaft. The motor and encoder were contained in a sealed streamlined housing, mounted to the force balance via a $30 \mathrm{~cm}$ aluminium strut. A propeller drive shaft was passed through the casing 
using a sealed bearing and connected to the motor output via an Oldham coupling (figure $5 \mathrm{C}$ ).

The motor power was controlled using pulse-width modulation (PWM), with all control and data acquisition performed in the LabView environment. Each propeller was driven from $10-100 \%$ PWM duty cycle, increasing in $10 \%$ increments. For every measurement thrust and rpm data were recorded for 10 seconds and averaged.

\section{A. Results and Analysis}

The results of three propeller tests are shown in figures 5D-F, with thrust curves shown for tests with 2 and 3 blade variants. The results show a close agreement with QPROP predictions across the three propellers presented.

Three-blade propellers were found to generate more thrust than a two-blade propeller with identical blade dimensions as expected, with an additional blade increasing the resistance load on the propeller as well as the lift produced by the blades. This increases the torque load at equilibrium for the motor-propeller system, resulting in higher current draw and thrust compared to two-blade propellers. As the motor used has a relatively high design torque, the system was generally well-matched across the range of propellers tested, allowing a large range of speeds to be tested until the motor reached its maximum output torque.

The computational predictions were found to be a close fit to the experimental data and show that the simulations provide an accurate representation of the actual system. A possible source of the minor discrepancy between the theoretical estimate and the measured data is the effect of significantly higher hydrodynamic forces acting on the blade underwater causing slight deformation near the tips. This flexibility was not accounted for in the QPROP simulations. Nevertheless, the experimental results were found to match the simulations closely, indicating that any effect caused by this deformation was not significant. Overall efficiency of the system cannot be concluded from the experiments as only static tests were conducted, meaning that $\eta_{P}=0$. However, other investigations in moving flowfields have also shown QPROP to be quite accurate at prediction of propeller flows at similar Reynolds numbers [14], [15], [16].

This confirms the results from section III that using an aerial propulsion system directly in off-design conditions will result in highly inefficient propulsion in water, in addition to damagingly high current draws for motors not designed to sustain high torque loads. In seeking a compromise between aerial and aquatic performance, the torque requirements to achieve desirable aquatic propulsion would result in a large reduction of thrust in air. As the operating rpm and torque is an equilibrium point based on the motor-propeller combination, a significant improvement in performance can be achieved by using a more flexible system that is capable of altering one of these variables to suit the operating medium.

\section{Aerial-Aquatic Locomotion}

From the propellers investigated, it is clear that using the same motor-propeller combination for multimodal loco- motion will be highly inefficient in at least one medium. This problem could be addressed to some extent by variable pitch propellers, but the mechanical complexity makes this challenging to implement for small scale vehicles. A more straightforward alternative is the use of two separate propulsion systems, optimised independently. However, the vehicle would have to carry the weight of an unused system at any point in its mission, and the inactive propulsor may also incur drag penalties.

Because the problem is not that aerial propellers are necessarily inefficient underwater, but that motors are poorly matched, the use of a variable transmission to ensure good matching is sensible. However, this again may be difficult to implement at the small scale, and requires a system for changing gear between media. Rather than employ an actuated gearbox, which incurs a weight and complexity penalty, we propose that reversing motor direction is a simple and lightweight means of controlling a two-speed gearbox for an aerial-aquatic robot.

To investigate the efficacy of a transmission system, we have used QPROP to compute an ideal transmission for an aerial propulsion system operating underwater. In table I, we list several propellers and compute the gear reduction which maximises efficiency underwater, if the $40 \mathrm{~W}$ brushless motor described in section III is used as a drive. Across the range of propellers simulated, it can be seen that using the optimal gearing gives an order of magnitude increase in efficiency underwater. This comparison once again shows the significance of motor-propeller matching in efficiency. Specifically, large gains can be made by adjusting the torque characteristics of the motor, while varying the diameter and number of blades within the design range contributes much less to performance. Under optimal gearing, the underwater efficiency is capable of obtaining a similar range as the aerial efficiency. This means that the system is able to produce significantly more thrust underwater, whilst also drawing a smaller amount of power. The power required underwater is also of a similar magnitude to that required in air, showing that underwater locomotion will not place any strain on the electronics of the vehicle.

\section{A. Gearbox Mechanism}

The simplest way to control the gearbox is to use the drive shaft to automatically engage the gearbox when operating in one direction (water mode) and disengage the gearbox in the other direction (air mode or direct drive), avoiding the complexity of a mechanical gear change, and the need for additional actuators. However, in both modes the output needs to be spinning in the same direction as the propeller is unchanged. In order to achieve this, the gearbox must reverse the direction of the output when engaged, which is done using a planetary gearset in fixed-carrier mode (figure 6AD). A second epicyclic stage in fixed-ring mode is added after the first to achieve the full reduction required, while preserving the original direction change. Both the output and input driveshaft to the gearbox are connected to the propeller using separate sprag clutches, which permit rotation in only 
TABLE I: Aerial-aquatic performance of different propellers being driven by a 40W brushless motor (unthrottled): For each propeller, we show the forward velocity $(v)$ at which the motor-propeller system achieves maximum efficiency. Aquatic performance without variable transmission is compared to the use of a calculated optimal gearing which maximises total efficiency, $\eta_{T}$, increasing thrust $(T)$ while greatly reducing electrical input power, $P_{i}$.

\begin{tabular}{|c|c|c|c|c|c|c|c|c|c|c|c|c|c|c|c|c|}
\hline \multirow{2}{*}{$\begin{array}{c}\text { Propeller } \\
\text { D x Pitch }(\mathrm{mm})\end{array}$} & \multicolumn{5}{|c|}{ Air, Direct Drive } & \multicolumn{5}{|c|}{ Underwater, Direct Drive } & \multicolumn{6}{|c|}{ Underwater, Optimal Gearbox } \\
\hline & $\eta_{T}$ & $\eta_{P}$ & $T(\mathrm{~N})$ & $V(\mathrm{~m} / \mathrm{s})$ & $P_{i}(\mathrm{~W})$ & $\eta_{T}$ & $\eta_{P}$ & $T(\mathrm{~N})$ & $v(\mathrm{~m} / \mathrm{s})$ & $P_{i}(\mathrm{~W})$ & Gearing & $\eta_{T}$ & $\eta_{P}$ & $T(\mathrm{~N})$ & $V(\mathrm{~m} / \mathrm{s})$ & $P_{i}(\mathrm{~W})$ \\
\hline $152 \times 76$ & 346 & ( & 1.0 & 1.1 & 32 & .023 & the & 3.8 & 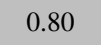 & 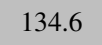 & . & 0. & 0.492 & 0 & 1 & 26.3 \\
\hline $152 \times 76$ (3-bla & 402 & 0.574 & 10 & 124 & 31.3 & 0.031 & 0 & 3. & 1 & 0 & 10. & 0.409 & 0568 & 8.6 & 1.2 & 24.2 \\
\hline $152 \times$ & 404 & 0.606 & 1.1 & 14.0 & 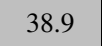 & .024 & 0 & 3.2 & 1.0 & .4 & $: 1$ & 0.426 & 0.594 & 0.5 & . & 25.3 \\
\hline $152 \mathrm{x}$ & 5 & 0.7 & 85 & 16 & 32.2 & 42 & 0 & 2 & 2.2 & 131.5 & $11.2: 1$ & 0. & 07 & 77 & 1.8 & 24.4 \\
\hline 178 & 425 & 0.619 & 1.1 & 13 & 34.5 & 028 & 0.514 & 3.4 & 1.1 & 34.2 & 1 & 0.4 & 0.608 & 8.90 & 1.2 & 25.1 \\
\hline $178 \times 8$ & 3 & 0. & 1.2 & 12.8 & 42.1 & 0 & 0 & 3.1 & 0.86 & & & $\mathbf{0}$ & 0.558 & 9.5 & 1.1 & 26.2 \\
\hline 178 & 71 & 0.592 & 1.3 & 13.1 & 46.3 & 0.019 & 0.509 & 3.5 & 0.74 & .7 & $: 1$ & 0.413 & 0.5 & 9.5 & .1 & 25.4 \\
\hline $178 \times 102$ (3-blade) & 0.310 & 0.536 & 1.4 & 11.8 & 54.7 & 0.013 & 0.439 & 3.2 & 0.58 & 137.8 & $15.3: 1$ & 0.369 & 0.515 & 10.0 & 0.96 & 25.9 \\
\hline
\end{tabular}
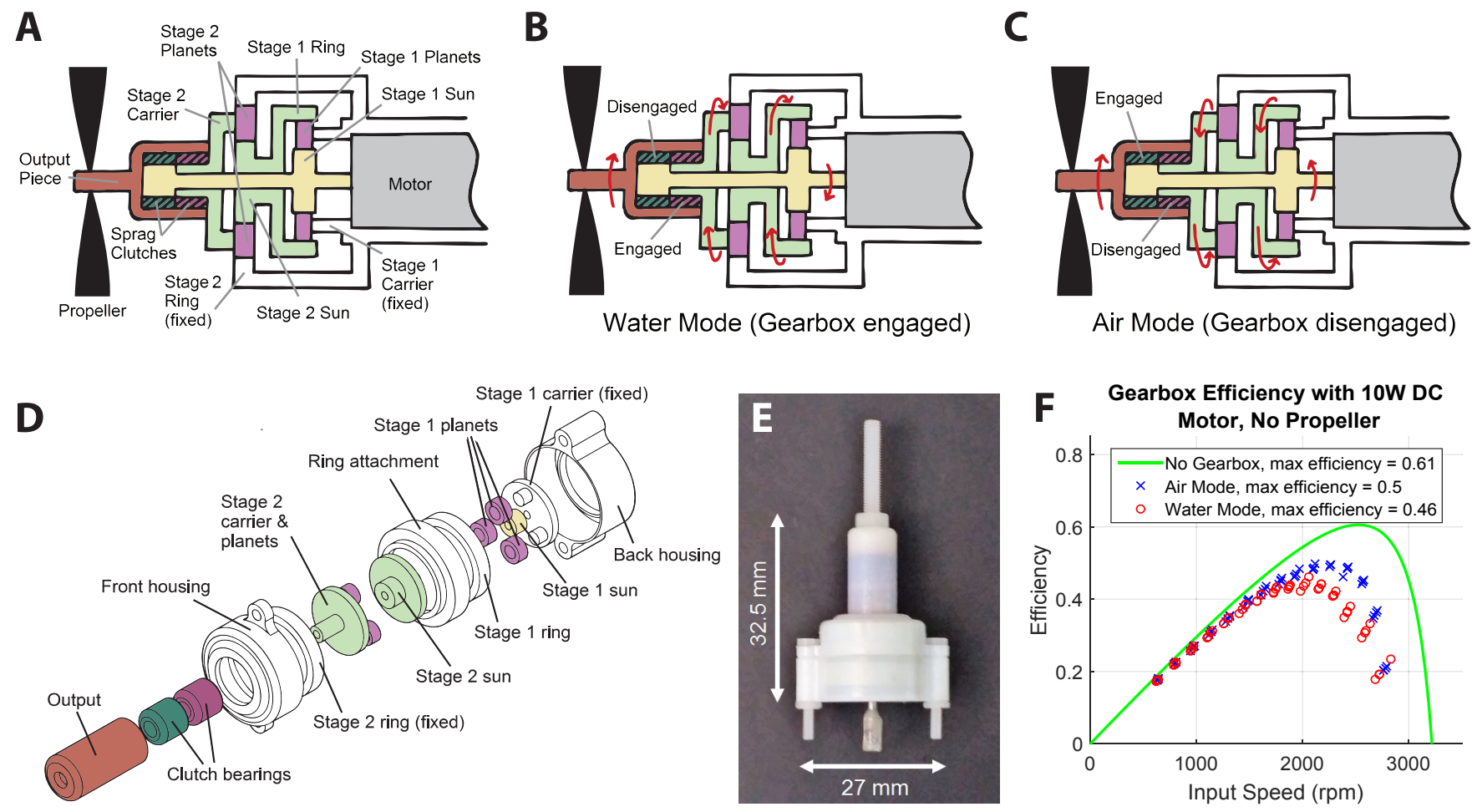

Fig. 6: A-C: The two operational modes of the gearbox determined by the direction of the motor shaft. D: Exploded view of the developed gearbox. E: Manufactured prototype of the gearbox. F: Efficiency data for fabricated gearbox being powered by a $10 \mathrm{~W}$ DC motor.

one direction. Because the output of the gearbox spins at a lower speed than the driveshaft, the two move relative to each other and are selectively decoupled from the propeller by the clutches. This means that while the gearbox can be driven by the motor in both directions, the propeller spins with the input when the motor rotates in one direction and with the gearbox output when it rotates in the other.

\section{B. Prototype}

A gearbox prototype was produced, an exploded view of which can be seen in figure 6D, showing the two planetary gear stages and two sprag clutches. The gearbox weighs 12 grams, is $27 \mathrm{~mm}$ wide, and $32.5 \mathrm{~mm}$ long (figure 6E). Miniature steel gears were used for the transmission, while the gear housing and output connection were 3D printed using a Connex Objet 350 printer. Using a $152 \times 102 \mathrm{~mm}$ propeller, our analysis (section $\mathrm{V}$ table I) gives an optimal gearing of 12.1:1, and as a result our gearbox is designed to obtain a gear reduction of 12.7:1, with a slight difference due to the availability of appropriately sized gears. This gearbox was run across a range of speeds (using PWM) by a $10 \mathrm{~W}$ DC motor (Pololu 10:1 HPCB6V), and by measuring the 

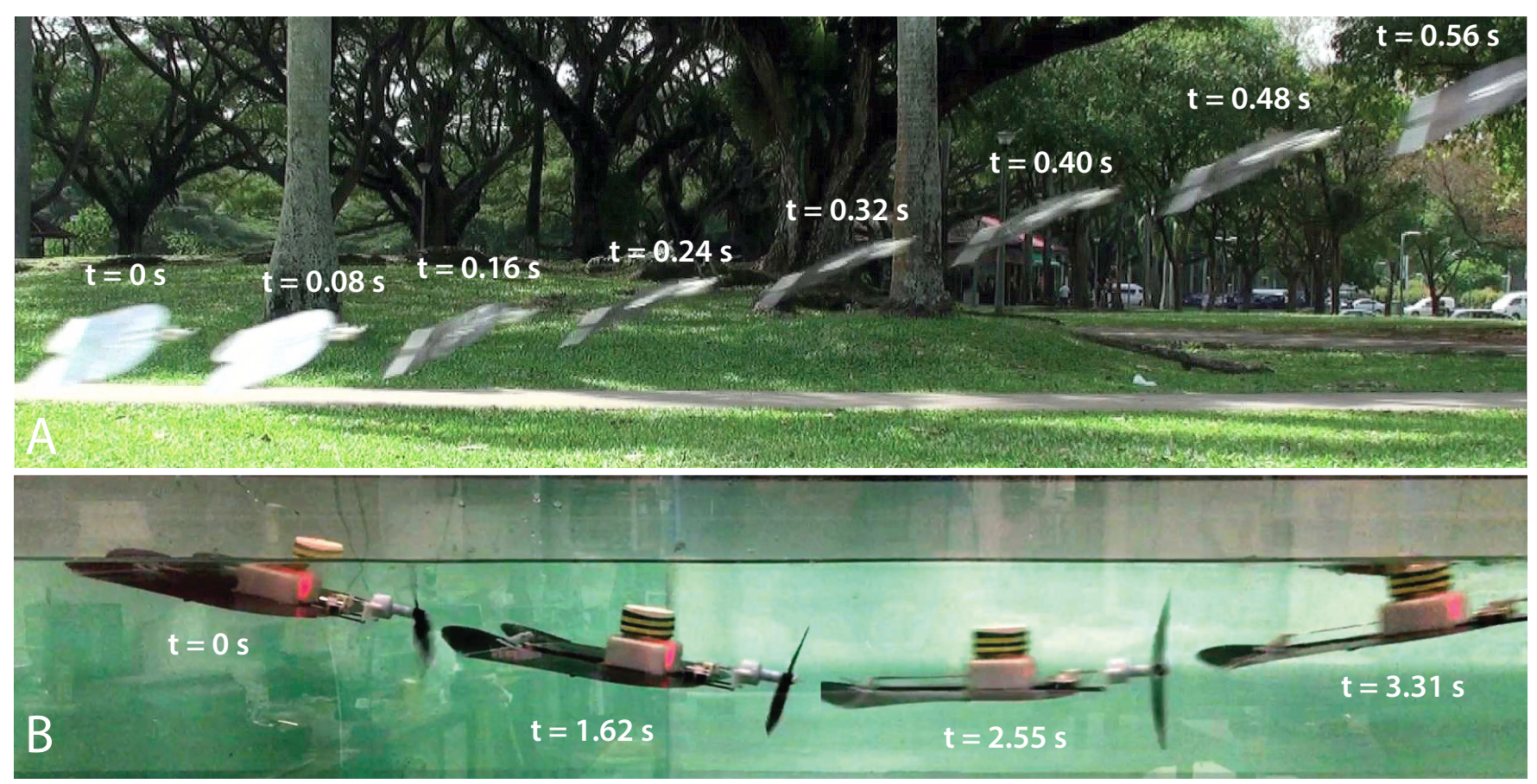

Fig. 7: Timelapse images from video footage of the aerial-aquatic gearbox propelling a robot in both air and water, with times annotated to show speed. (A) Aerial-aquatic robot flying in air, with gearbox driven forward. (B) Aerial-aquatic robot moving underwater, with gearbox driven in reverse. In water a plastic fairing is added to waterproof the electronics and provide buoyancy.

difference between the measured speeds of the motor with and without the gearbox attached, we were able to calculate the resistance torque the gearbox creates at a given input speed (using equation 4), and thereby the loss of efficiency (figure 6F). The tests showed that the maximum achievable efficiency of the motor was reduced by $18 \%$ when operating with the gearbox disengaged (air mode) and by $24 \%$ with the gearbox engaged (water mode). The friction losses are similar as gear train rotates in both modes, but in air mode, the input shaft rotates in the opposite direction to the stage 2 carrier (figure 6B) so the increased relative motion results in higher friction. The friction in the drive is slightly higher than anticipated, due mainly to the poor alignment achieved using 3D-printed plastic to house the transmission, which can be readily improved in a future prototype.

To demonstrate the device's functionality in air and water, the gearbox was then integrated into a custom made miniature radio controlled robot. The uncambered aircraft wing is made from a $0.25 \mathrm{~mm}$ carbon fibre plate, reinforced with undirectional strips, and has a wingspan of $280 \mathrm{~mm}$. A radio receiver with integrated servos is used to control two elevons for steering, and the total system weighs 50 grams (figure 2). Figure 7] shows a timelapse image of the robot in flight and travelling in water. The robot was able to fly in air, despite the additional gearbox friction reducing the available power. When used underwater, the electronics were protected by a seal plastic fairing, which also provided buoyancy. Driven in reverse, the gearbox was effective underwater, and the vehicle was able to dive and surface using control surfaces (figure 7). Dive depth was not a priority for these tests and was limited by the poor penetration of $\mathrm{GHz}$ radio into water, indicating that either additional communication or some level of autonomy may be required for an effective aerial aquatic robot.

The most significant limitation of this demonstrator as an aerial aquatic vehicle is its inability to transition between the two modes of locomotion, as the robot has insufficient power for takeoff from water. However, solutions to this problem have recently been presented [17], [18], which will be integrated with a future prototype. The plastic fairing to protect the components underwater created too large a drag and weight penalty in air, but can be easily replaced by waterproof coatings such as Parylene-C.

\section{CONCLUSION}

A prototype system for reconciling the differences between aerial and aquatic propulsion was demonstrated through the integration of a specially designed gearbox. The novel use of a two stage planetary system with paired one-way clutch bearings allowed the mode of the gearbox to be controlled with no additional actuators by simply reversing the direction of the motor. The final prototype showed promising results in testing, allowing the aerial propulsion system to operate at a the appropriate speed underwater. As the gearbox is an independent add-on, no additional controls are required on the vehicle beyond directional control of the existing motor, and the simplicity of the design allows it to be easily integrated into other aerial-aquatic systems. 
To fully realise the underwater travel capabilities of an AquaMAV, additional work is required to give the vehicle the ability to transition between different locomotion modes, by integrating sufficient propulsive power for take off from water, and sufficient structural strength to allow dives into water at aerial flight speed. Attention must also be given to appropriate buoyancy and dynamic stability in water as well as a solution to communication at depth. The performance of the motor-gearbox coupled system can be further improved by using better machined parts with higher precision. Future work can also include the study of using water cooling to improve the performance range of the motor underwater. Nevertheless, the findings above and working solution presented are a strong starting point in the implementation of underwater propulsion solutions for small aerial robots, which would greatly expand the mobility of unmanned vehicles, and allow for many new applications.

\section{ACKNOWLEDGEMENTS}

The authors would like to thank the Imperial College technical staff, in particular Roland Hutchins and Mark Grant, for their indispensable help with experimental work and fabrication. This project was funded by the UK Engineering and Physical Sciences Research Council.

\section{REFERENCES}

[1] R. Siddall and M. Kovac, "Launching the aquamav: bioinspired design for aerial-aquatic robotic platforms," Bioinspiration \& Biomimetics, vol. 9, no. 3, 2014.

[2] _ _ "Fast aquatic escape with a jet thruster," IEEE/ASME Transactions on Mechatronics, 2016.

[3] M. Drela, "Qprop: Propeller/windmill analysis and design," 2007.

[4] R. Lock, S. Burgess, and R. Vaidyanathan, "Multi-modal locomotion: from animal to application," Bioinspiration \& biomimetics, vol. 9, no. 1, p. 011001, 2013.

[5] K. Low, T. Hu, S. Mohammed, J. Tangorra, and M. Kovac, "Perspectives on biologically inspired hybrid and multi-modal locomotion," Bioinspiration \& biomimetics, vol. 10, no. 2, p. 020301, 2015.

[6] X. Yang, T. Wang, J. Liang, G. Yao, and M. Liu, "Survey on the novel hybrid aquatic-aerial amphibious aircraft: Aquatic unmanned aerial vehicle (aquauav)," Progress in Aerospace Sciences, vol. 74, pp. 131-151, 2015.

[7] J. Izraelevitz and M. Triantafyllou, "A novel degree of freedom in flapping wings shows promise for a dual aerial/aquatic vehicle propulsor," arXiv preprint arXiv:1412.3843, 2014.

[8] R. J. Lock, R. Vaidyanathan, S. C. Burgess, and J. Loveless, "Development of a biologically inspired multi-modal wing model for aerialaquatic robotic vehicles through empirical and numerical modelling of the common guillemot, uria aalge," Bioinspiration \& Biomimetics, vol. 5, no. 4, p. 046001, 2010.

[9] Y. Chen, E. F. Helbling, N. Gravish, K. Ma, and R. J. Wood, "Hybrid aerial and aquatic locomotion in an at-scale robotic insect," in 2015 IEEE/RSJ International Conference on Intelligent Robots and Systems (IROS), 2015.

[10] H. Alzu'bi, O. Akinsanya, N. Kaja, I. Mansour, and O. Rawashdeh, "Evaluation of an aerial quadcopter power-plant for underwater operation," in 2015 10th International Symposium on Mechatronics and its Applications (ISMA). IEEE, 8-10 Dec. 2015 2015, p. 4 pp.

[11] M. M. Maia, P. Soni, and F. J. Diez, "Demonstration of an aerial and submersible vehicle capable of flight and underwater navigation with seamless air-water transition," CoRR, vol. abs/1507.01932, 2015.

[12] E. E. Larrabee, Practical Design of Minimum Induced Loss Propellers. SAE, 1979.

[13] R. Siddall, A. Ortega Ancel, and M. Kovac, "Wind and water tunnel testing of a morphing aquatic micro air vehicle," Interface Focus, In Press 2017.
[14] T. Sinnige and L. L. Veldhuis, "Pylon trailing edge blowing effects on the performance and noise production of a pusher propeller."

[15] H. Youngren and M. Chang, "Test, analysis and design of propeller propulsion systems for mavs," in 49th AIAA Aerospace Sciences Meeting including the New Horizons Forum and Aerospace Exposition, AIAA, vol. 876, 2011.

[16] S. B. Heinzen and A. Gopalarathnam, "Passively varying pitch propeller for small uas," 2010.

[17] R. Siddall and M. Kovac, "A water jet thruster for an aquatic micro air vehicle," in 2015 IEEE International Conference on Robotics and Automation (ICRA), 2015, p. 3979.

[18] R. Siddall, G. Kennedy, and M. Kovac, "Explosive propulsion strategies for aquatic take off in robotics," in 2015 International Symposium on Robotics Research (ISRR), 2015. 